\title{
Iron Homeostasis and Ferritin in Sepsis-Associated Kidney Injury
}

\author{
Kayla McCullough Subhashini Bolisetty \\ Department of Medicine, Division of Nephrology, University of Alabama at Birmingham, \\ Birmingham, AL, USA
}

\section{Keywords}

Ferritin · Iron · Sepsis-associated kidney injury · Cytokine storm

\begin{abstract}
Sepsis associated acute kidney injury (SA-AKI) is a common clinical syndrome that occurs among hospitalized patients and significantly impacts mortality. Furthermore, survival after sepsis is intricately dependent on recovery of kidney function. In this review, we discuss the role of iron imbalance in mediating the pathogenic events during sepsis. Intracellular ferritin serves as a repository for iron and prevents ironmediated injury and may limit the availability of iron to pathogens. Circulating levels of ferritin also increase during sepsis and often correlate with severity of sepsis. Herein, we examine preclinical and clinical data and discuss recent findings that suggest immunomodulatory roles for ferritin. We also discuss the possible mechanistic roles for ferritin in mitigating the pathogenic sequelae of sepsis and highlight current gaps in knowledge.

๑) 2020 S. Karger AG, Basel
\end{abstract}

Contribution from the AKI and CRRT 2020 Symposium at the 25th International Conference on Advances in Critical Care Nephrology, Manchester Grand Hyatt, San Diego, CA, USA, February 24-27, 2020. This symposium was supported in part by the NIDDK-funded University of Alabama at Birmingham-University of California San Diego O'Brien Center for Acute Kidney Injury Research (P30DK079337).

\section{Introduction}

Sepsis is defined as a "life-threatening dysfunction caused by dysregulated host response to infection." Sepsis is the leading cause of acute kidney injury (AKI) with 45$70 \%$ of all AKI considered to be sepsis associated-AKI (SA-AKI). SA-AKI significantly impacts mortality and recovery from AKI and is intricately linked to survival [1]. Increase in mortality during the early acute phase may be attributed to the inflammatory cytokine storm, oxidative stress, mitochondrial dysfunction, and disrupted iron homeostasis. This review will examine and discuss the potential effects of iron imbalance and ferritin, an intracellular iron storage protein, in the pathogenesis of sepsis.

\section{Impact of Regulating Iron Levels during Sepsis}

Iron plays a major role in many metabolic reactions, and therefore both the host and pathogen compete for iron. In an effort to starve the pathogens of iron, the liver synthesizes and releases hepcidin. Hepcidin prevents egress of iron from cells by inducing degradation of ferroportin, an iron exporter. This causes hypoferremia, a rapid decline in levels of iron in circulation, which may prevent bacterial proliferation and dampen oxidative stress by preventing iron-mediated free radical generation. Based on this physiological response, several studies

$\begin{aligned} & \text { karger@karger.com } \\ & \text { www.karger.com/nef }\end{aligned}$
Karger ${ }^{\prime /}$

Subhashini Bolisetty

Department of Medicine, University of Alabama at Birmingham ZRB 6201720 2nd Ave. S

Birmingham, AL 35294 (USA)

sbolisetty@uabmc.edu 
Table 1. Effects of iron modulation during sepsis-AKI

\begin{tabular}{|c|c|c|c|c|c|}
\hline Species & Treatment & Time of treatment & Outcome & Results & PMID \\
\hline \multicolumn{6}{|c|}{ Iron supplementation } \\
\hline Humans & $\begin{array}{l}12.5-62.5 \text { mg of intravenous } \\
\text { iron (ferric gluconate) }\end{array}$ & Weekly & Reduced mortality & Positive & 25462819 \\
\hline Humans & $\begin{array}{l}125 \mathrm{mg} / \text { day of sodium ferric } \\
\text { gluconate }\end{array}$ & Post-SA-AKI for 2-8 days & $\begin{array}{l}\text { No adverse effect in recovery of AKI } \\
\text { or mortality }\end{array}$ & No change & 26801821 \\
\hline Mice & $\begin{array}{l}2 \mathrm{mg} \text { of intravenous iron } \mathrm{Fe}^{3+} \\
\text { in theform of Fe sucrose }\end{array}$ & Time of sepsis & $\begin{array}{l}\text { Increase in ROS, TNF- } \alpha \text {, and } \\
\text { mortality }\end{array}$ & Negative & 15149323 \\
\hline \multicolumn{6}{|c|}{ Iron chelation } \\
\hline Mice & $50-100 \mu g$ of hepcidin & Pre- or post-LPS or CLP & $\begin{array}{l}\text { Pre-Tx reduced SA-AKI and } \\
\text { mortality. Post-Tx reduced SA-AKI } \\
\text { and bacteremia }\end{array}$ & Positive & 31244655 \\
\hline Mice & $40 \mathrm{mg} / \mathrm{kg}$ DIBI & $\begin{array}{l}\text { Post-colon ascendens stent peritonitis } \\
\text { (CASP) } 3 \text { or } 13 \mathrm{~h}\end{array}$ & Reduced bacteremia & Positive & 26235905 \\
\hline \multirow[t]{2}{*}{ Rats } & \multirow{2}{*}{$\begin{array}{l}20 \mathrm{mg} / \mathrm{kg} \text { of } N \text {-acetylcysteine } \\
(\mathrm{NAC}) \text { and } \mathrm{DFO}\end{array}$} & NAC: 3-24 h after CLP, subcutaneously & \multirow{2}{*}{$\begin{array}{l}\text { Reduced septic shock by decreasing } \\
\text { ROS, neutrophil infiltration, } \\
\text { mitochondrial dysfunction and } \\
\text { mortality }\end{array}$} & \multirow[t]{2}{*}{ Positive } & \multirow[t]{2}{*}{14758146} \\
\hline & & DFO: 3-24 h after CLP, subcutaneously & & & \\
\hline Rats & $40 \mathrm{mg} / \mathrm{kg}$ of DFX & $\begin{array}{l}20 \mathrm{mg} / \mathrm{kg} \text { subcutaneously immediately } \\
\text { post-CLP, } 20 \mathrm{mg} / \mathrm{kg} \text { subcutaneously } \\
\text { again } 6 \mathrm{~h} \text { post-CLP }\end{array}$ & Reduced mortality & Positive & 15037222 \\
\hline
\end{tabular}

SA-AKI, sepsis associated acute kidney injury; TNF- $\alpha$, tumor necrosis factor-alpha; CLP, cecal ligation and puncture; LPS, lipopolysaccharide.

have investigated the effects of iron chelation or supplementation during sepsis and have produced conflicting results (Table 1). Iron supplementation was associated with a decrease in all-cause mortality and a reduced sepsis-related mortality rate in a cohort of dialysis patients [2]. Conversely, intravenous iron supplementation of patients with SA-AKI had no significant effect on recovery of AKI or mortality [3]. However, in contrast, another study showed that parenteral iron supplementation given at the time of sepsis resulted in worse outcomes due to an increase in oxidative stress and tumor necrosis factor-alpha (TNF- $\alpha$ ) [4]. High levels of serum iron were independently associated with an increase in 90-day mortality rates in patients with sepsis [5]. These data indicate that iron supplementation could exacerbate SA-AKI and align with previous findings that iron starvation may augment host defense mechanisms [6]. In a preclinical study, iron chelation via desferoxamine (DFX), an iron chelator, resulted in increased survival of rats and was associated with an increase in renal expression of the proapoptotic protein, Bax, during sepsis [7]. Interestingly, pretreatment of mice with hepcidin also protects against SA-AKI and mortality. Hepcidin mediated macrophage iron retention and consequently increased $\mathrm{FtH}$ expression [8].
Several studies have demonstrated that iron chelation prior to induction of AKI is protective (Table 1); however, the effect of iron chelators following established renal injury is not well studied. Also, iron restriction using agents such as DFO or hepcidin may be most effective against siderophilic bacteria and extracellular pathogens but may not be protective against nonsiderophilic or intracellular pathogens, including Mycobacterium tuberculosis. It should also be recognized that while acute iron chelation may be beneficial against bacteremia and oxidative stress, prolonged iron chelation may negatively impact the host by inducing anemia and subsequently organ dysfunction. Therefore, the potential of iron chelation during SA-AKI requires further investigation.

\section{Elevation of Circulating Ferritin during Sepsis}

Ferritin is a ubiquitously expressed spherical protein that comprises 24 subunits of heavy $(\mathrm{FtH})$ and light $(\mathrm{FtL})$ chains. $\mathrm{FtH}$ is a ferroxidase which converts ferrous iron into ferric iron, which is subsequently stored within the ferritin shell. FtL does not have ferroxidase activity, and its presumed function was limited to iron nucleation. In- 
tracellular tissue ferritin can store up to 4,500 atoms of iron in a safe and bioavailable form. However, circulating (serum) ferritin is predominantly composed of FtL and is low in iron content [9]. While the antioxidant and iron repository role of tissue ferritin is extensively studied, the function of circulating ferritin is still unclear. In the clinic, serum ferritin is routinely used to evaluate body iron stores and inflammation; a decrease in circulating ferritin levels is indicative of low iron, whereas an increase is indicative of iron overload. However, ferritin levels also increase during inflammation and confound the clinical utility of ferritin in iron management.

Ferritin is elevated during sepsis, and hyperferritinemia often correlates with severity of sepsis [10]. Additionally, treatment of hyperferritinemic patients with an interleukin-1 receptor antagonist reduced sepsis-induced mortality from 66 to 35\%, suggesting that elevation of ferritin was associated with inflammation [11]. While the preponderance of studies establish a correlation between elevated ferritin and worse outcomes, few studies demonstrate that a failure to increase ferritin was also associated with mortality [12]. Additionally, serum ferritin levels predicted renal function recovery in patients with AKI [13]. It should be noted that these clinical studies were associative and do not necessarily establish a causal relationship with severity of AKI or mortality. Of note, FtL hyperferritinemia, an autosomal dominant syndrome, is associated with increased intracellular FtL and elevated serum ferritin levels. Also, serum ferritin levels are significantly higher in transgenic mice heterozygous for the FtH gene. Interestingly, such elevation in FtL (in both humans and rodents) is benign and is not associated with increased inflammation, suggesting a contradiction in the presumed association of serum ferritin with inflammation. Therefore, the significance of elevated ferritin during infection and inflammatory conditions warrants further investigation.

\section{Ferritin in Preclinical Models of Sepsis-AKI}

As described in the previous section, circulating ferritin levels are often elevated during sepsis. However, the contributory role of ferritin in mediating the pathogenic events during sepsis could not be established in these clinical studies. In this connection, Lipiński et al. [14] demonstrated that administration of tissue ferritin (derived from horse spleen, murine liver, or bovine spleen) significantly protected mice against a lethal Escherichia coli infection. Interestingly, iron saturation of these tissue fer-

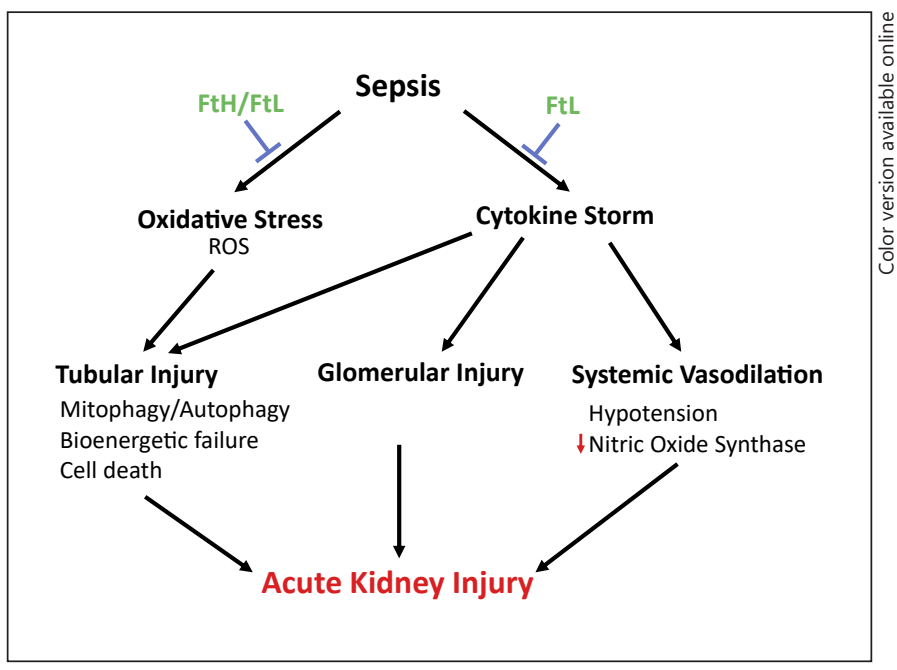

Fig. 1. Role of ferritin in sepsis associated-AKI. Ferritin is an essential iron sequestering, antioxidant protein that protects against sepsis-associated kidney injury. Serum ferritin, specifically ferritin light chain, prevents the cytokine storm and consequently SAAKI. SA-AKI, sepsis associated acute kidney injury.

ritins varied from 11 to $46 \%$ but did not influence mortality, suggesting that the protective effects of ferritin were independent of iron loading. They also found a dose- and time-dependent effect of ferritin, with maximal protection derived from administration of ferritin $24 \mathrm{~h}$ prior to infection. Similarly, Weis et al. [15] recapitulated these findings in a mouse model of polymicrobial sepsis using apoferritin that is completely devoid of iron. These intriguing findings suggest that the protective effects of ferritin may not be limited to iron sequestration.

Host survival is dependent on resistance to infections; however, data show that tolerance is equally important to preserving host metabolism and promoting survival. Ramos et al. [16] showed that FtH expression in renal proximal tubules is essential to establish tolerance against Plasmodium spp.-induced AKI. FtH also sustained gluconeogenesis in hepatocytes during sepsis and promoted tolerance [15]. These studies emphasize the role of FtH in mediating tolerance against infections.

To better understand the role of iron metabolism and ferritin on sepsis-induced inflammation and AKI, we generated transgenic mice with targeted deletion of FtH in myeloid cells (macrophages and neutrophils). Macrophages are central mediators of iron recycling and systemic iron homeostasis. Also, iron storage requires $\mathrm{FtH}$ and, therefore, deletion of $\mathrm{FtH}$ in macrophages prevents intracellular iron sequestration. Additionally, previous reports suggested that circulating ferritin was predominantly derived 
from macrophages. Intriguingly, we found that myeloid $\mathrm{FtH}$ deletion conferred significant protection against sepsis-AKI and mortality in 2 models of sepsis, cecal ligation and puncture (CLP), or lipopolysaccharide (LPS) endotoxemia [17]. Corroborating previous reports, wild-type mice had significantly high levels of pro- and anti-inflammatory cytokines (IL-6, TNF- $\alpha$, IL-1 $\beta$, IL-4, and IL-10) following sepsis induction. In contrast, loss of $\mathrm{FtH}$ from the myeloid compartment abrogated the cytokine storm. Sepsis caused leukopenia in both the animal groups and loss of $\mathrm{FtH}$ did not influence the number or proportion of immune cells in circulation or kidneys. Interestingly, while CLP increased hepcidin levels in both groups of mice, FtH-deficient mice had significantly lower levels of hepcidin compared to wild-type mice, suggesting that hepcidin-induced hypoferremia is not the dominant protective mechanism in the absence of FtH. As expected, sepsis led to an increase in circulating ferritin levels. However, loss of $\mathrm{FtH}$ was associated with significantly elevated circulating ferritin levels even in animals that underwent sham surgery. Importantly, the increase in serum ferritin did not lower serum iron levels or affect transferrin saturation, suggesting that circulating ferritin does not influence extracellular iron metabolism during sepsis. Myeloid FtH-deficient mice expressed significantly higher levels of FtL in macrophages and subsequently in circulation. To determine whether the elevated ferritin levels conferred resistance to myeloid $\mathrm{FtH}$-deficient mice, recombinant FtL was administered to wild-type mice prior to sepsis induction. Intriguingly, FtL administration significantly dampened the hyperinflammatory response, reduced organ dysfunction, and promoted survival (Fig. 1).

In order to delineate the mechanism of FtL-mediated immunomodulatory effects, we examined the transcriptomic profile of blood leukocytes following sepsis induction. As expected, the inflammatory response and associated pathways were significantly upregulated in wild-type leukocytes with a dominant NFkB footprint. These findings were also recapitulated in vitro using bone-marrowderived macrophages. Additionally, activation of mitogen-activated protein kinase pathways, including c-Jun $\mathrm{N}$-terminal kinase (JNK) and extracellular signal-related kinase (ERK), was heightened in wild-type macrophages [17]. Both loss of FtH and overexpression of FtL were associated with a reduction in NFkB activation and subsequent inflammatory response. These data underscore an important role for ferritin light chain and serum ferritin in mediating a regulated and measured inflammatory response to sepsis to prevent hyperinflammation and kidney injury.

Ferritin Light Chain in Sepsis-AKI

\section{Conclusion}

Iron is a prerequisite for many cellular processes that enable survival, oxidative stress, and inflammation. Therefore, acute chelation of iron to limit availability to pathogens is tempting, but prolonged iron chelation may lead to detrimental consequences, including metabolic dysfunction, anemia, and organ injury. Levels of circulating ferritin, predominantly FtL, are often elevated during sepsis and correlate with severity of disease. Our recent findings shed light on this evolutionarily conserved protein and identify FtL as a potent immunomodulatory effector. Collectively, we speculate that $\mathrm{FtH}$ is an essential iron sequestering antioxidant that promotes tolerance. On the other hand, FtL may mediate immunoregulation and prevent sepsis-AKI (Fig. 1). These findings also underscore the underappreciated role of ferritin in preventing AKI during sepsis. Further investigation into the functions of ferritins during sepsis may enable development of new treatments for SA-AKI that could alleviate the significant burden of sepsis-induced morbidity and mortality and substantial health care expenditures.

\section{Conflict of Interest Statement}

The authors have no conflicts of interest to declare.

\section{Funding Sources}

This work was supported in part by a NIH grant (DK103931 to S.B.) and an ASN grant (Carl W. Gottschalk Award to S.B.).

References

1 Peerapornratana S, Manrique-Caballero CL, Gómez H, Kellum JA. Acute kidney injury from sepsis: current concepts, epidemiology, pathophysiology, prevention and treatment. Kidney Int. 2019 Nov;96(5):1083-99.

2 Zitt E, Sturm G, Kronenberg F, Neyer U, Knoll F, Lhotta K, et al. Iron supplementation and mortality in incident dialysis patients: an observational study. PLoS One. 2014;9(12): e114144.

3 Clark BA, Osadchuk L, John J, Culver T, Marcus R. Effect of intravenous iron on outcomes of acute kidney injury. Transfusion. 2016 Apr; 56(4):933-7.

4 Zager RA, Johnson AC, Hanson SY. Parenteral iron therapy exacerbates experimental sepsis. Kidney Int. 2004 Jun;65(6):2108-12.

5 Lan P, Pan KH, Wang SJ, Shi QC, Yu YX, Fu $\mathrm{Y}$, et al. High serum iron level is associated with increased mortality in patients with sepsis. Sci Rep. 2018 Jul 23;8(1):11072. 
6 Ganz T Iron in innate immunity: starve the invaders. Curr Opin Immunol. 2009 Feb; 21(1):63-7.

7 Messaris E, Antonakis PT, Memos N, Chatzigianni E, Leandros E, Konstadoulakis MM. Deferoxamine administration in septic animals: improved survival and altered apoptotic gene expression. Int Immunopharmacol. 2004 Mar;4(3):455-9.

8 Scindia Y, Wlazlo E, Leeds J, Loi V, Ledesma J, Cechova S, et al. Protective role of hepcidin in polymicrobial sepsis and acute kidney injury. Front Pharmacol. 2019;10:615.

9 Wang W, Knovich MA, Coffman LG, Torti FM, Torti SV. Serum ferritin: past, present and future. Biochim Biophys Acta. 2010 Aug; 1800(8):760-9.
10 Kernan KF, Carcillo JA. Hyperferritinemia and inflammation. Int Immunol. 2017 Nov 1; 29(9):401-9.

11 Kyriazopoulou E, Leventogiannis K, NorrbyTeglund A, Dimopoulos G, Pantazi A, Orfanos SE, et al. Macrophage activation-like syndrome: an immunological entity associated with rapid progression to death in sepsis. BMC Med. 2017 Sep 18;15(1):172.

12 Carcillo JA, Simon DW, Podd BS. How we manage hyperferritinemic sepsis-related multiple organ dysfunction syndrome/macrophage activation syndrome/secondary hemophagocytic lymphohistiocytosis histiocytosis. Pediatr Crit Care Med. 2015 Jul;16(6): 598-600.

13 Dimitrijevic ZM, Salinger-Martinovic SS, Jankovic RJ, Mitic BP. Elevated serum ferritin levels are predictive of renal function recovery among patients with acute kidney injury. Tohoku J Exp Med. 2019 Jun;248(2):63-71.
14 Lipiński P, Jarzabek Z, Broniek S, Zagulski T. Protective effect of tissue ferritins in experimental Escherichia coli infection of mice in vivo. Int J Exp Pathol. 1991 Dec;72(6):623-30.

15 Weis S, Carlos AR, Moita MR, Singh S, Blankenhaus B, Cardoso S, et al. Metabolic adaptation establishes disease tolerance to sepsis. Cell. 2017 Jun 15;169(7):1263-e14.

16 Jeney V, Ramos S, Bergman ML, Bechmann I, Tischer J, Ferreira A, et al. Control of disease tolerance to malaria by nitric oxide and carbon monoxide. Cell Rep. 2019 Mar 19;8(1): 126-36.

17 Zarjou A, Black LM, McCullough KR, Hull TD, Esman SK, Boddu R, et al. Ferritin light chain confers protection against sepsis-induced inflammation and organ injury. Front Immunol. 2019;10:131. 